\title{
ON REGULARIZATION PARAMETER FOR LO-SPARSE COVARIANCE FITTING BASED DOA ESTIMATION
}

\author{
Alice Delmer ${ }^{\star \dagger} \quad$ Anne Ferréol ${ }^{\star \dagger} \quad$ Pascal Larzabal ${ }^{\dagger}$ \\ * Thales, Gennevilliers, France \\ ${ }^{\dagger}$ SATIE, CNRS, Université Paris-Saclay, Cachan, France
}

\begin{abstract}
In sparse DOA estimation methods, the regularization parameter $\lambda$ is generally empirically tuned. In this paper, we provide a statistical method allowing to estimate an admissible interval where $\lambda$ must be chosen. This work is conducted in the case of an Uniform Circular Array, well known for its $\theta$ invariant performances, and vectorized covariance matrix observation. In the recent work [1], it is shown that the equivalence between the $\ell_{0}$-constrained problem and the corresponding regularized one is obtained for $\lambda$ belonging to a given interval. This interval is conditional to an observation. The purpose of this work is to generalize this result for stochastic observations, providing so an interval $I$ of $\lambda$ valid in all scenarios for an UCA. This interval is not data dependent. Simulation results validate the proposed approach.
\end{abstract}

Index Terms - DOA estimation, sparse regularization, regularization parameter

\section{INTRODUCTION}

Recently, the sparse representation framework introduced in DOA estimation literature has shown numerous interests in comparison with conventional methods such as beamforming, Capon's method and MUSIC. We can cite for example the cases of coherent sources [2] [3], less sensors than sources, or close sources [4]. The mathematical foundation of sparse estimation has first been developed for Single Measurement Vector (SMV) model. The aim is to recover a sparse vector $\gamma_{0}$ from a noisy observation $\mathbf{y}=\mathbf{B} \gamma_{0}+\Delta \mathbf{r}$, requiring to solve the following constrained problem:

$$
\min _{\boldsymbol{\gamma}}\|\mathbf{B} \boldsymbol{\gamma}-\mathbf{y}\|_{2}^{2}, \quad \text { subject to }\|\boldsymbol{\gamma}\|_{0} \leq M,
$$

where the $\ell_{0}$ pseudo-norm (usually referred to as $\ell_{0}$-norm) is defined as $\|\mathbf{x}\|_{0}=\operatorname{Card}\left\{g \in\{1, \ldots, G\}: x_{g} \neq 0\right\}, x_{g}$ is the $g$ th components of vector $\mathbf{x}$, and $M$ the sparsity level. Another possible formulation is the following regularized form:

$$
\min _{\gamma}\left\{J_{\ell_{0}}(\lambda, \gamma)=\frac{1}{2}\|\mathbf{B} \gamma-\mathbf{y}\|_{2}^{2}+\lambda\|\gamma\|_{0}\right\},
$$

where the regularization parameter $\lambda>0$ balances the relative importance between data fidelity and sparsity.
In the field of DOA estimation, the SMV model directly applies on a single array snapshot. Multi Measurement Vector (MMV) schemes [5] have then been applied on several snapshots to improve estimation. In order to reduce the computational cost of MMV based schemes without losing any information contained in the snapshots collection, [6] proposes to use the eigenvectors of the signal subspace, thus reducing the size of the observation matrix. More recently, vectorized covariance matrix modeling has been introduced to transform a MMV problem into SMV [7] [8] [9], leading again to a decrease in the complexity, providing an easier implementation, and allowing to use the more advanced schemes of SMV. This is thus the framework used in this article.

Problems (1) and (2) are NP-hard but numerous methods have been proposed to find a good approximate solution. Convex $\ell_{1}$ relaxation is probably the most common approach, although sufficient conditions to recover a sparse signal with $\ell_{1}$ minimization [10] are usually too restrictive in practical applications. Greedy algorithms also have major drawbacks when dealing with highly correlated columns in the dictionary B. Iterative Hard Thresholding (IHT) [11] [12] presents the drawback of converging to one of the numerous local minima of the $\ell_{0}$-regularized criterion. Recently, the sparse community has shown the interest of bounded penalties as approximations of the $\ell_{0}$-norm in the regularized criterion [13] [14] [15] [16] [17] [18]. Surprisingly, to the best of our knowledge, these recent works and more generally the $\ell_{0}$-like framework are not yet very popular in array signal processing literature, and never been used for sparse DOA estimation based on vectorized covariance matrix. This article aims to fill this lack.

For that, we focus on the sparse $\ell_{0}$ minimization using, as a SMV observation, the vectorized covariance matrix under the assumption that the sources are uncorrelated. The recently introduced CEL0 penalty [16] will be used to approximate the $\ell_{0}$-norm. It has been proved that this penalty suppresses some local minima of the $\ell_{0}$-regularized criterion, without changing the position and value of the global minima. The regularization parameter $\lambda$ plays a decisive role in the solution obtained when minimizing $J_{\ell_{0}}$, but it is usually empirically tuned : we analyze this role more deeply in this article. 


\section{MODEL AND PROBLEM FORMULATION}

\subsection{Array signal modeling}

Let us consider $M$ narrowband sources impinging an array of $N$ antennas from angles $\tilde{\theta}_{m}, m=1 \ldots M$. The output signal $\mathbf{x}(t) \in \mathbb{C}^{N}$ is a linear combination of the array responses (or steering vectors) $\mathbf{a}\left(\tilde{\theta}_{m}\right)$ of the DOAs plus an additive white Gaussian noise of zero mean and variance $\sigma_{n}^{2}$ : $\mathbf{x}(t)=\sum_{m=1}^{M} \mathbf{a}\left(\tilde{\theta}_{m}\right) \tilde{s}_{m}(t)+\mathbf{n}(t)$. Supposing the sources are on a predefined known grid containing $G$ candidate directions, the output signal can thus be modeled using the sparse representation framework as :

$$
\mathbf{x}(t)=\mathbf{A} \mathbf{s}(t)+\mathbf{n}(t) .
$$

$\mathbf{A} \in \mathbb{C}^{N \times G}$ is a calibration table containing the steering vectors for all directions in the grid $\mathbf{A}=\left[\mathbf{a}\left(\theta_{1}\right) \ldots \mathbf{a}\left(\theta_{G}\right)\right]$ and the vector $\mathbf{s}(t) \in \mathbf{C}^{G}$ is sparse with $M$ non-zero entries corresponding to the sources signals.

\subsection{SMV observation from MMV measurement : the vectorized covariance matrix model}

Under the single array snapshot model (3), sparse DOA estimation consists of the retrieval of the indices of nonzero components in $\mathbf{s}(t)$, which indicate the directions via the corresponding columns of $\mathbf{A}$. Using multiple snapshots for the estimation under the MMV framework increases the accuracy but also the computational cost. As an alternative and to work under the SMV framework using multiple snapshots, a vectorized covariance matrix based scheme is proposed.

In this article, it is assumed that the sources are uncorrelated. The covariance matrix $\mathbf{R}_{x x}=E\left[\mathbf{x}(t) \mathbf{x}^{H}(t)\right]$ is thus equal to $\mathbf{R}_{x x}=\sum_{m=1}^{M} \mathbf{a}\left(\tilde{\theta}_{m}\right) \mathbf{a}^{H}\left(\tilde{\theta}_{m}\right) \tilde{\gamma}_{m}+\sigma_{n}^{2} \mathbf{I}_{N}$, where $\mathbf{I}_{N}$ is the square identity matrix and $\tilde{\gamma}_{m}$ is the power of the $g$ thsource. The vectorized covariance matrix $\mathbf{r}=\operatorname{vec}\left(\mathbf{R}_{x x}\right)$ is thus of the form $\mathbf{r}=\sum_{m=1}^{M} \mathbf{b}\left(\tilde{\theta}_{m}\right) \tilde{\gamma}_{m}+\sigma_{n}^{2} \operatorname{vec}\left(\mathbf{I}_{N}\right)$, where $\mathbf{b}(\theta)=\mathbf{a}^{*}(\theta) \otimes \mathbf{a}(\theta), \mathbf{a}^{*}$ is the conjugate of $\mathbf{a}$, and $\otimes$ is the Kronecker product. We then have

$$
\mathbf{r}=\mathbf{B} \boldsymbol{\gamma}_{0}+\sigma_{n}^{2} \operatorname{vec}\left(\mathbf{I}_{N}\right),
$$

where $\mathbf{B} \in \mathbf{C}^{N^{2} \times G}$ is defined as $\mathbf{B}=\left[\mathbf{b}\left(\theta_{1}\right), \ldots, \mathbf{b}\left(\theta_{G}\right)\right]$, and $\gamma_{0}=\left[\gamma_{1} \ldots \gamma_{G}\right]^{T}$. Vector $\gamma_{0} \in \mathbb{R}^{G}$ is $M$-sparse, the nonzero components represent the power of the true incoming sources and their indices indicate the corresponding directions.

For $K$ snapshots, the covariance matrix can be estimated by $\hat{\mathbf{R}}_{x x}=1 / K \sum_{t=1}^{K} \mathbf{x}(t) \mathbf{x}^{H}(t)$. Lets note $\hat{\mathbf{r}}=\operatorname{vec}\left(\hat{\mathbf{R}}_{x x}\right)$. In this article, the observation vector $\mathbf{y} \in \mathbb{C}^{N^{2}}$ is defined as

$$
\mathbf{y}=\hat{\mathbf{r}}-\sigma_{n}^{2} \operatorname{vec}\left(\mathbf{I}_{N}\right)=\mathbf{B} \boldsymbol{\gamma}_{0}+\Delta \mathbf{r}
$$

$\Delta \mathbf{r}$ represents the noise : the observation, based on the vectorized covariance matrix of multiple snapshots, corresponds to a SMV model of size $N^{2}$. Within this article, we will suppose that $\sigma_{n}^{2}$ is known, although it might be estimated in practice.

\subsection{Problem formulation}

The purpose of this original work is to analyze a sparse $\ell_{0}$ DOA estimation scheme based on the unique observation vector $\mathbf{y}$. The goal is to find the solution of (1) through the minimization of (2). The adjustment of the regularization parameter $\lambda$ is then crucial. After our preliminary work in [19], the aim of this paper is to evaluate an admissible range for $\lambda$, allowing to automatically tune the parameter. This will be done thanks to a theoretical statistical analysis, within the context of $M=2$ impinging sources.

\section{AN ADMISSIBLE RANGE FOR $\lambda$ : THEORETICAL ANALYSIS}

\subsection{Equivalence between the constrained and regular- ized problems for a given observation $y$}

Our approach is based on the work [1]. For a single observation $\mathbf{y}$, it gives a condition on the regularization parameter $\lambda$ so that the optimal solution of the minimization of the criterion $J_{\ell_{0}}$ in (2) is also the solution of the constrained problem with sparsity $M(1)$. Define $c_{k}$ as the optimal value of the least-square residual for a vector with sparsity $k$ :

$$
c_{k}=\inf \left\{\|\mathbf{B} \boldsymbol{\gamma}-\mathbf{y}\|_{2}^{2} \mid \gamma \in \mathbb{C}^{G} \text { and }\|\boldsymbol{\gamma}\|_{0} \leq k\right\} .
$$

The optimal sets of problems (1) and (2) coincide for a given observation $\mathbf{y}$ if and only if $\lambda_{M}^{-}<\lambda<\lambda_{M}^{+}$[1] with:

$$
\begin{aligned}
& \lambda_{M}^{-}=\max _{k}\left\{\frac{c_{M}-c_{k}}{2(k-M)} \mid M<k \leq N^{2}\right\}, \\
& \lambda_{M}^{+}=\min _{k}\left\{\frac{c_{k}-c_{M}}{2(M-k)} \mid 0 \leq k<M\right\} .
\end{aligned}
$$

Let's note that the optimal value $c_{k}$ (6) can be computed as :

$$
\begin{gathered}
c_{k}=\min _{\boldsymbol{\Phi}_{\mathbf{k}}}\left(c\left(\boldsymbol{\Phi}_{\mathbf{k}}\right)\right) \\
c\left(\boldsymbol{\Phi}_{\mathbf{k}}\right)=\mathbf{y}^{H} \boldsymbol{\Pi}_{n}\left(\boldsymbol{\Phi}_{\mathbf{k}}\right) \mathbf{y},
\end{gathered}
$$

with $\Pi_{n}$ the noise projector $\boldsymbol{\Pi}_{n}\left(\boldsymbol{\Phi}_{\mathbf{k}}\right)=\mathbf{I}-\boldsymbol{\Pi}\left(\boldsymbol{\Phi}_{\mathbf{k}}\right)$ associated with the signal projector $\Pi$, computed as $\Pi\left(\boldsymbol{\Phi}_{\mathbf{k}}\right)=$ $\mathbf{B}\left(\boldsymbol{\Phi}_{\mathbf{k}}\right)\left[\mathbf{B}\left(\boldsymbol{\Phi}_{\mathbf{k}}\right)^{H} \mathbf{B}\left(\boldsymbol{\Phi}_{\mathbf{k}}\right)\right]^{-1} \mathbf{B}\left(\boldsymbol{\Phi}_{\mathbf{k}}\right)^{H}$, where $\mathbf{B}\left(\boldsymbol{\Phi}_{\mathbf{k}}\right)$ is the submatrix of $\mathbf{B}$ containing only the steering vectors corresponding to angles in the set $\boldsymbol{\Phi}_{\mathbf{k}}=\left\{\varphi_{1}, \ldots, \varphi_{k}\right\}$.

\subsection{Statistical distributions of $\lambda_{2}^{+}$and $\lambda_{2}^{-}$}

In this article, the focus is on the scenario $M=2$ well separated sources. In this context, we are interested in the parameters $\lambda_{2}^{+}$and $\lambda_{2}^{-}$. Nikolova [1] defined an admissible interval for $\lambda, I=] \lambda_{2}^{-} ; \lambda_{2}^{+}$[ conditionally to an observation y. Computation of those parameters requires to solve the different $k$-constrained problems in a combinatorial fashion, which is of course not a possible option for a practical implementation. 
Preliminary simulations in [19] exhibits potential existence of an interval $I$ admissible for stochastic observations. In this paper, we theoretically investigate this interval in the case of stochastic realizations of the vectorized covariance matrix in a given scenario (DOA, SNR). In order to evaluate this interval off-line, we provide the statistics of $\lambda_{2}^{-}$and $\lambda_{2}^{+}$, thanks to the derivation of the statistics of the observations $\mathbf{y}$.

\subsubsection{Distribution of the observation $\mathbf{y}$}

It is known that $\hat{\mathbf{R}}_{x x}=\mathbf{R}_{x x}+\Delta \mathbf{R}_{x x}$ with $K \Delta \mathbf{R}_{x x}$ follows a Wishart distribution [20]. Then $\mathbf{y}=\mathbf{r}-\sigma_{n}^{2} \operatorname{vec}\left(\mathbf{I}_{N}\right)+\Delta \mathbf{r}$ follows asymptotically a complex normal multivariate distribution $\mathcal{C N}(\boldsymbol{\mu}, \boldsymbol{\Gamma}, \mathbf{C})$ with:

$$
\begin{gathered}
\boldsymbol{\mu} \hat{=} E[\mathbf{y}]=\mathbf{r}-\sigma_{n}^{2} \operatorname{vec}\left(\mathbf{I}_{N}\right) \\
\boldsymbol{\Gamma} \hat{=} E\left[(\mathbf{y}-\boldsymbol{\mu})(\mathbf{y}-\boldsymbol{\mu})^{H}\right]=\frac{1}{K} \mathbf{R}_{x x}^{T} \otimes \mathbf{R}_{x x} \\
\mathbf{C} \hat{=} E\left[(\mathbf{y}-\boldsymbol{\mu})(\mathbf{y}-\boldsymbol{\mu})^{T}\right]=E\left[\Delta \mathbf{r} \Delta \mathbf{r}^{T}\right]
\end{gathered}
$$

The relation matrix $\mathbf{C}$ is computed by block: $E\left[\Delta \mathbf{r}_{\mathbf{i}} \Delta \mathbf{r}_{\mathbf{j}}{ }^{T}\right]=$ $1 / K \cdot \mathbf{r}_{\mathbf{j}} \mathbf{r}_{\mathbf{i}}{ }^{T}$, where $\Delta \mathbf{r}_{\mathbf{i}}$ is the $i$-th $(N \times 1)$ subvector of the $\left(N^{2} \times 1\right)$ vector $\Delta \mathbf{r}$, and $\mathbf{r}_{\mathbf{i}}$ the $i$-th $(N \times 1)$ subvector of the vector $\mathbf{r}$. Note that $\boldsymbol{\Gamma}$ is hermitian and $\mathbf{C}$ is symmetric.

\subsubsection{Statistics of $\lambda_{2}^{+}$}

Under weak conditions, not developed here for lack of space, and assuming no outliers, it can be shown that:

$$
\begin{gathered}
c_{1} \approx \min \left\{c\left(\tilde{\theta}_{1}\right), c\left(\tilde{\theta}_{2}\right)\right\}, \quad c_{2} \approx c\left(\left\{\tilde{\theta}_{1}, \tilde{\theta}_{2}\right\}\right) \\
\lambda_{2}^{+} \approx \frac{c_{1}-c_{2}}{2} \approx \frac{1}{2} \min \left\{Q_{12}(\mathbf{y}), Q_{21}(\mathbf{y})\right\},
\end{gathered}
$$

with, for $\{i, j\}=\{1,2\}, i \neq j$ :

$$
\begin{aligned}
Q_{i j}(\mathbf{y}) & =c\left(\tilde{\theta}_{i}\right)-c\left(\left\{\tilde{\theta}_{i}, \tilde{\theta}_{j}\right\}\right) \\
& =\mathbf{y}^{H}\left(\boldsymbol{\Pi}_{n}\left(\tilde{\theta}_{i}\right)-\mathbf{\Pi}_{n}\left(\left\{\tilde{\theta}_{i}, \tilde{\theta}_{j}\right\}\right)\right) \mathbf{y}
\end{aligned}
$$

Calculations on projectors leads to:

$\boldsymbol{\Pi}_{n}\left(\boldsymbol{\Phi}_{\mathbf{k}}\right)-\boldsymbol{\Pi}_{n}\left(\left\{\boldsymbol{\Phi}_{\mathbf{k}}, \Phi_{k+1}\right\}\right)=P\left(\boldsymbol{\Pi}_{n}\left(\boldsymbol{\Phi}_{\mathbf{k}}\right) \mathbf{B}\left(\Phi_{k+1}\right)\right)$ with $P(\mathbf{x})=\mathbf{x}\left[\mathbf{x}^{H} \mathbf{x}\right]^{-1} \mathbf{x}^{H}$. Thus we have:

$$
Q_{i j}(\mathbf{y})=\mathbf{y}^{H} P\left(\boldsymbol{\Pi}_{n}\left(\tilde{\theta}_{i}\right) \mathbf{B}\left(\tilde{\theta}_{j}\right)\right) \mathbf{y}
$$

$Q_{12}(\mathbf{y})$ and $Q_{21}(\mathbf{y})$ are hermitian quadratic forms in noncentral normal variables. An hermitian quadratic form $Q(\mathbf{y})=\mathbf{y}^{H} \mathbf{Q y}$, with $\mathbf{y} \sim \mathcal{C N}(\boldsymbol{\mu}, \boldsymbol{\Gamma}, \mathbf{C})$ can be expressed as a real quadratic form $Q(\mathbf{y})=\mathbf{y}_{\mathbf{R}}{ }^{T} \mathbf{Q}_{\mathbf{R}} \mathbf{y}_{\mathbf{R}}$ with [21]:

$$
\begin{gathered}
\mathbf{y}_{\mathbf{R}}=\left[\Re(\mathbf{y})^{T}, \Im(\mathbf{y})^{T}\right]^{T}, \\
\mathbf{y}_{\mathbf{R}} \sim \mathcal{N}\left(\boldsymbol{\mu}_{\boldsymbol{R}}, \boldsymbol{\Sigma}\right) \\
\boldsymbol{\mu}_{\boldsymbol{R}}=\left[\Re(\boldsymbol{\mu})^{T}, \Im(\boldsymbol{\mu})^{T}\right]^{T} \\
\boldsymbol{\Sigma}=\frac{1}{2}\left(\begin{array}{cc}
\Re(\boldsymbol{\Gamma}+\mathbf{C}) & \Im(-\boldsymbol{\Gamma}+\mathbf{C}) \\
\Im(\boldsymbol{\Gamma}+\mathbf{C}) & \Re(\boldsymbol{\Gamma}-\mathbf{C})
\end{array}\right) \\
\mathbf{Q}_{\mathbf{R}}=\left(\begin{array}{cc}
\left(\mathbf{Q}+\mathbf{Q}^{T}\right) / 2 & i\left(\mathbf{Q}-\mathbf{Q}^{T}\right) / 2 \\
i\left(\mathbf{Q}^{T}-\mathbf{Q}\right) / 2 & \left(\mathbf{Q}+\mathbf{Q}^{T}\right) / 2
\end{array}\right)
\end{gathered}
$$

Under this form, the distribution of $Q(\mathbf{y})$ can be approximated by a non-central chi-square distribution $\chi_{l}^{2}(\delta)$ so that it matches the third-order moment and has the best match with the fourth-order moment [22]. The mean, standard deviation, skewness and kurtosis of $Q(\mathbf{y})$ are given by:

$$
\mu_{Q}=s_{1}, \sigma_{Q}=\sqrt{2 s_{2}}, \beta_{1}=\sqrt{8} b_{1}, \beta_{2}=12 b_{2}
$$

with $s_{k}=\operatorname{Tr}\left(\left(\mathbf{Q}_{\mathbf{R}} \boldsymbol{\Sigma}\right)^{k}\right)+k \boldsymbol{\mu}_{\boldsymbol{R}}^{T}\left(\mathbf{Q}_{\mathbf{R}} \boldsymbol{\Sigma}\right)^{k-1} \mathbf{Q}_{\mathbf{R}} \boldsymbol{\mu}_{\boldsymbol{R}}, b_{1}=$ $s_{3} / s_{2}^{3 / 2}$ and $b_{2}=s_{4} / s_{2}^{2}$. The non-centrality parameter $\delta$ and the number of degrees of freedom $l$ are thus computed as [22]:

- if $b_{1}^{2}>b_{2}, \delta=b_{1} a^{3}-a^{2}$ and $l=a^{2}-2 \delta$ with $a=$ $1 /\left(b_{1}-\sqrt{b_{1}^{2}-b_{2}}\right)$,

- if $b_{1}^{2} \leq b_{2}, \delta=0$ and $l=1 / b_{1}^{2}$.

The distributions of $\lambda_{2}^{+}$can be approximated as the distribution of the min of two non-central chi-square distribution. To the best of our knowledge, there is no closed-form expression of such distribution yet. Using Eq. (19) to (23), samples of the approximate distributions of $Q_{12}(\mathbf{y})$ and $Q_{21}(\mathbf{y})$ can be generated numerically in order to obtain the distribution of the minimum (15). Fig. 1(a) represents the cumulative distribution function of $\lambda_{2}^{+}$and its approximations. The three curves for $\lambda_{2}^{+}$are almost confounded.

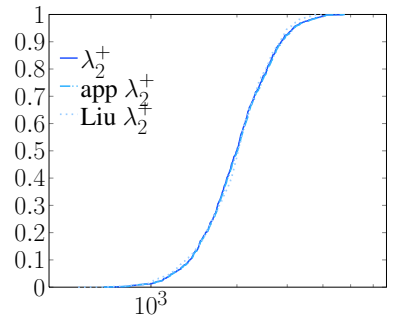

(a) $\lambda_{2}^{+}$

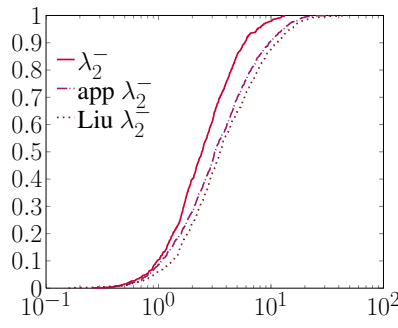

(b) $\lambda_{2}^{-}$
Fig. 1. Cumulative distribution function of $\lambda_{2}^{+}$and $\lambda_{2}^{-}$. Solid lines: obtained in a combinatorial fashion. Dash dotted lines: simulated with approximations (14) and (24). Dotted lines: Liu's non-central chi-square approximations [22].

\subsubsection{Statistics of $\lambda_{2}^{-}$}

It can be shown that:

$$
\begin{gathered}
c_{3} \approx \min _{\theta} c\left(\left\{\tilde{\theta}_{1}, \tilde{\theta}_{2}, \theta\right\}\right) \\
\lambda_{2}^{-} \approx \frac{c_{2}-c_{3}}{2} \approx \frac{1}{2} \max _{\theta \neq \tilde{\theta}_{1}, \tilde{\theta}_{2}} Q_{3}(\mathbf{y}, \theta)
\end{gathered}
$$

where $Q_{3}(\mathbf{y}, \theta)$ is defined by

$$
Q_{3}(\mathbf{y}, \theta)=\mathbf{y}^{H} P\left(\boldsymbol{\Pi}_{n}\left(\left\{\tilde{\theta}_{1}, \tilde{\theta}_{2}\right\}\right) \mathbf{B}(\theta)\right) \mathbf{y} .
$$

The distribution of $\lambda_{2}^{-}$is thus the distribution of the maximum of $G-2$ correlated quadratic forms whose distributions can be approximated as non-central chi-square. To the best of our 
knowledge, there is no closed-form expression of such distribution. In order to obtain this distribution, correlated data of (26) are numerically generated. Lets note that, for an off-line estimation of $\lambda$, the computational cost decreases drastically in comparison with the initial combinatorial problem (8) (6).

\section{NUMERICAL RESULTS}

An Uniform Circular Array (UCA) of $N=7$ antennas and radius $d=\frac{\lambda_{0}}{2}$, where $\lambda_{0}$ is the wavelength, is considered. The vectorized covariance matrix is computed with $K=50$ snapshots. The $M=2$ impinging sources originate from DOA $\tilde{\theta}_{1}=0^{\circ}$ and variable $\tilde{\theta}_{2}$.

\subsection{Interval $I$ for stochastic observations (given DOAs)}

In this simulation, $\tilde{\theta}_{2}=68^{\circ}$ and both sources have the same power, with $S N R=8 \mathrm{~dB}$. Equations (15) and (25) along with Liu's approximation of the quadratic forms [22] are used to simulate the distribution of $\lambda_{2}^{+}$and $\lambda_{2}^{-}$from 1000 samples in this setup (Fig. 2, top). 1000 Monte Carlo simulations are performed to solve (2) for values of $\lambda$ between $10^{-1}$ and $10^{4}$. The minimization of the $\ell_{0}$-regularized criterion is performed by the use of an IRL1 algorithm applied to Continuous Exact$\ell_{0}$ (CEL0) penalty [23]. Root-Mean Square Error (RMSE) is computed, in degrees, between the estimated directions and the true DOAs (Fig. 2, middle). As expected for an UCA, RMSE is the same for both sources. For the calculation of this RMSE, only good detections are considered : a false detection corresponds to an outlier outside the beamwidth. The percentage of errors is represented in Fig. 2 (bottom). This simulation clearly shows the existence of an interval $I$ for $\lambda$ without outliers, i.e no error, and corresponding to minimal RMSE. As expected, this interval $I=[10 ; 300]$ roughly corresponds to $\left[\max \lambda_{2}^{-}, \min \lambda_{2}^{+}\right]$of the computed histograms. It is important to note that those histograms are obtained prior to the processing.
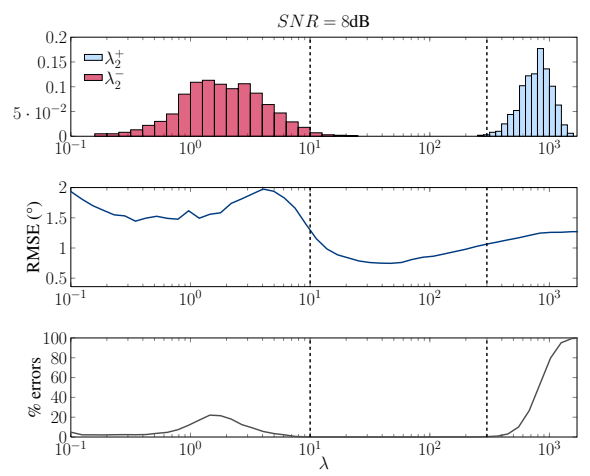

Fig. 2. $\tilde{\theta}_{1}=0^{\circ}, \tilde{\theta}_{2}=68^{\circ}$ and $S N R=8 \mathrm{~dB}$ for both sources. Top: simulated pdf of $\lambda_{2}^{+}$and $\lambda_{2}^{-}$. RMSE in degrees (middle) of the solution obtained by IRL1-CEL0, and percentage of errors (bottom) as a function of $\lambda$.

\subsection{Versatility of interval $I$}

In simulations not presented here, the interval $I$ was studied depending on the spacing between sources, for a given SNR. It appears that the min of the distribution of $\lambda_{2}^{+}$does not depend on the DOAs for well separated sources. The max of $\lambda_{2}^{-}$depends however on the spatial correlation between the two sources. For a practical implementation, it is possible to take, as a minimal bound for $I$, the worst case scenario.

Other simulations were carried out for sources of different power. In this scenario, $\tilde{\theta}_{1}=0^{\circ}, \tilde{\theta}_{2}=68^{\circ}, S N R_{1}=10 \mathrm{~dB}$ while the SNR of the second source increases. It appears that the distribution of $\lambda_{2}^{+}$remains quite stationary while the distribution of $\lambda_{2}^{-}$varies depending on the SNR of the most powerful source. Fig. 3 represents the histograms, RMSE and percentage of error for $S N R_{2}=22 \mathrm{~dB}$. At this SNR, the histogram of $\lambda_{2}^{-}$starts to overlap with the histogram of $\lambda_{2}^{+}$: the percentage of errors is minimal in the middle of the overlapping but it is not null, at $1.5 \%$. Deriving the statistics of $\lambda_{2}^{+}$ and $\lambda_{2}^{-}$can be used to determine the operating limits.
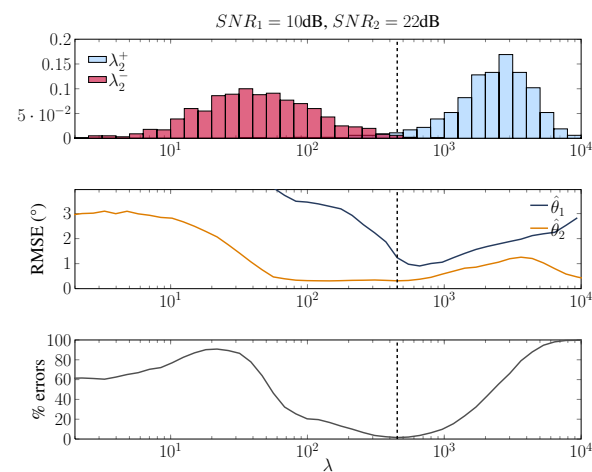

Fig. 3. $\tilde{\theta}_{1}=0^{\circ}, \tilde{\theta}_{2}=68^{\circ}, S N R_{1}=10 \mathrm{~dB}$ and $S N R_{2}=$ 22dB. Simulated pdf (top) of $\lambda_{2}^{+}$and $\lambda_{2}^{-}$. RMSE in degrees (middle) of the solution obtained by IRL1-CEL0, and percentage of errors (bottom) as a function of $\lambda$.

\section{CONCLUSION}

This work has concerned sparse $\ell_{0}$ estimation based on vectorized covariance matrix. We have proposed a versatile approach to soundly select the regularization parameter $\lambda$, contrasting with the widespread empirical choice. In the case of an UCA, we have shown the potential existence of an interval $I$ for $\lambda$ allowing a robust estimation of the DOAs. It is important to note that this interval is determined before the processing and is not data dependent. Ongoing work concerns now the parametric modelization of the extreme values of the distributions of $\lambda_{2}^{+}$and $\lambda_{2}^{-}$. This will give a tool to determine such an interval for any experimental system. 


\section{REFERENCES}

[1] Mila Nikolova, "Relationship between the optimal solutions of least squares regularized with L0-norm and constrained by k-sparsity," Applied and Computational Harmonic Analysis, vol. 41, no. 1, pp. 237-265, 2016.

[2] Amiri Parian Mahnaz and Ghofrani Sedigheh, "ML1,2MUSIC algorithm for DOA estimation of coherent sources," IET Signal Processing, vol. 11, no. 4, pp. 429436, 2017.

[3] Yuexian Wang, Xin Yang, Jian Xie, Ling Wang, and Brian W.H. Ng, "Sparsity-Inducing DOA Estimation of Coherent Signals under the Coexistence of Mutual Coupling and Nonuniform Noise," IEEE Access, vol. 7, pp. 40271-40278, 2019.

[4] Md Mashud Hyder and Kaushik Mahata, "Direction-ofArrival Estimation Using a Mixed 2;0 Norm Approximation," IEEE Transactions on Signal Processing, vol. 58, no. 9, 2010 .

[5] Shane F Cotter, Bhaskar D Rao, Kjersti Engan, and Kenneth Kreutz-Delgado, "Sparse Solutions to Linear Inverse Problems With Multiple Measurement Vectors," IEEE Transactions on Signal Processing, vol. 53, no. 7, 2005.

[6] Dmitry Malioutov, Müjdat Çetin, and Alan S Willsky, "A Sparse Signal Reconstruction Perspective for Source Localization With Sensor Arrays," IEEE Transactions on Signal Processing, vol. 53, no. 8, 2005.

[7] Joseph S. Picard and Anthony J. Weiss, "Direction finding of multiple emitters by spatial sparsity and linear programming," in 9th International Symposium on Communications and Information Technology, ISCIT, 2009.

[8] Z.Q He, Q.H. Liu, L.N. Jin, and S. Ouyang, "Low complexity method for DOA estimation using array covariance matrix sparse representation," Electronics Letters, vol. 49, pp. 228-230, 012013.

[9] Wei Cui, Tong Qian, and Jing Tian, "Enhanced covariances matrix sparse representation method for doa estimation," Electronics Letters, vol. 51, no. 16, pp. 12881290, 2015.

[10] Emmanuel J Candes, "The Restricted Isometry Property and Its Implications for Compressed Sensing," Comptes Rendus Mathématiques, vol. 346, no. 9, 2008.

[11] Thomas Blumensath and Mike E. Davies, "Iterative Thresholding for Sparse Approximations," Journal of Fourier Analysis and Applications, vol. 14, no. 5, pp. 629-654, 2008.
[12] Thomas Blumensath and Mike E. Davies, "Normalized iterative hard thresholding: Guaranteed stability and performance," IEEE Journal of Selected Topics in Signal Processing, vol. 4, pp. 298-309, 2010.

[13] Jianqing Fan and Runze Li, "Variable Selection via Nonconcave Penalized Likelihood and its Oracle Properties," Journal of the American Statistical Association, vol. 96, no. 456, pp. 1348-1360, 2001.

[14] Hosein Mohimani, Massoud Babaie-Zadeh, and Christian Jutten, "A fast approach for overcomplete sparse decomposition based on smoothed L0 norm," IEEE Transactions on Signal Processing, vol. 57, pp. 289301, 2009.

[15] Cun Hui Zhang, "Nearly unbiased variable selection under minimax concave penalty," Annals of Statistics, vol. 38, no. 2, pp. 894-942, 2010.

[16] Emmanuel Soubies, Laure Blanc-Féraud, and Gilles Aubert, "A Continuous Exact L0 penalty (CELO) for least squares regularized problem," SIAM J. Imaging Sciences, vol. 8, 2015.

[17] Marcus Carlsson, "On convexification/optimization of functionals including an 12-misfit term," arXiv preprint arXiv:1609.09378, 2016.

[18] Ivan Selesnick, "Sparse Regularization via Convex Analysis," IEEE Transactions on Signal Processing, vol. 65, no. 17, pp. 4481-4494, 2017.

[19] Alice Delmer, Anne Ferréol, and Pascal Larzabal, "Selection of regularization parameter in sparse inverse problems for doa estimation," to be published in : Journal of Physics: Conference Series, 2019.

[20] N. R. Goodman, "Statistical analysis based on a certain multivariate complex gaussian distribution (an introduction)," Ann. Math. Statist., vol. 34, no. 1, pp. 152-177, 031963.

[21] Ali Akbar Mohsenipour and Serge B. Provost, On the Distribution of Quadratic Expressions in Various Types of Random Vectors, Ph.D. thesis, 2012.

[22] Huan Liu, Yongqiang Tang, and Hao Helen Zhang, "A new chi-square approximation to the distribution of nonnegative definite quadratic forms in non-central normal variables," Computational Statistics and Data Analysis, vol. 53, no. 4, pp. 853-856, feb 2009.

[23] Simon Gazagnes, Emmanuel Soubies, and Laure BlancFéraud, "High density molecule localization for superresolution microscopy using cel0 based sparse approximation," in 14th International Symposium on Biomedical Imaging (ISBI 2017). IEEE, 2017, pp. 28-31. 